Original Article

\title{
Experimental Joint Immobilization and Remobilization in the Rats
}

Satoshi Kojima, RPT, MS ${ }^{1,2)^{*}}$, Masahiro Hoso, MD, PhD ${ }^{2)}$, Masanori Watanabe, RPT, PhD ${ }^{3)}$, Taro Matsuzaki, RPT, PhD ${ }^{2)}$, Itaru Hibino, RPT, PhD ${ }^{3)}$, Kentaro Sasaki, RPT, MS ${ }^{1)}$

1) Department of Physical Therapy, Faculty of Health Sciences, Kinjo University: 1200 Kasama-machi, Hakusan-city, Ishikawa 924-8511, Japan

2) Division of Health Sciences, Graduate School of Medical Science, Kanazawa University, Japan

3) Department of Physical Therapy, Faculty of Rehabilitation Sciences, Nagoya Gakuin University, Japan

\begin{abstract}
Purpose] The purpose of our study was to clarify temporal effects on restrictions to range of motion and the histopathological changes of joint components after joint immobilization in a rat knee-contracture model. [Subjects] Fifty-four male Wistar rats were randomly divided into two groups: a fixation group, and a control group. [Methods] In the fixation group, unilateral knee joints were immobilized at full flexion using a plaster cast for 4 weeks. At four weeks the animals were randomly divided into six subgroups, corresponding to the time of examination after cast removal: $0,4,8,16,24$, and 32 weeks. For comparison, control group animals of corresponding age were also examined. [Results] Although movement restrictions of the knee joint had completely recovered 6 weeks after the cast removal, cartilage and synovial membrane structures did not completely recover. [Conclusion] These findings have not previously been reported, and as they form an addition to the fundamental scientific foundations of physical therapy, further research must examine these findings from a variety of perspectives.

Key words: Contracture, Articular cartilage, Synovial membrane
\end{abstract}

(This article was submitted Nov. 20, 2013, and was accepted Jan. 7, 2014)

\section{INTRODUCTION}

Joint contracture causes functional disturbances in patients' activities of daily living, decreased movement ability, and impairs the joint function, with high incidence, ${ }^{1,2)}$. Therefore, preventing the development of joint contracture is a key goal of physical therapists and rehabilitation specialists. Unfortunately, medical professionals are still struggling to find definitive therapies for the prevention and management of joint contracture.

Although a number of factors can lead to the development of contractures ${ }^{3)}$, differentiating between them in a clinical setting can be difficult. In addition, different classifications are used by different specialists. Orthopedic surgeons generally classify contractures on the basis of changes causing restriction of the normal range of motion of a joint, a rigidity resulting from some extracapsular cause ${ }^{3-5}$. In physical therapy, classifying cases in terms of the area causing the condition has little practical purpose. Therefore, joint immobility causing restricted range of motion can be treated as a case of contracture ${ }^{2)}$.

*Corresponding author. Satoshi Kojima (E-mail: kojima@ kinjo.ac.jp)

(C2014 The Society of Physical Therapy Science. Published by IPEC Inc. This is an open-access article distributed under the terms of the Creative Commons Attribution Non-Commercial No Derivatives (by-ncnd) License $<$ http://creativecommons.org/licenses/by-nc-nd/3.0/> .
Changes in the cartilage and the synovial membrane have been reported as intracapsular joint sequelae resulting from contracture ${ }^{6-9)}$. To date, significant discrepancies exist among reports, and thus, the current state of research is inadequate for drawing meaningful conclusions. We previously clarified organic changes in the joint composition and structure resulting from contracture ${ }^{10,11)}$ : fibrosis of the cartilage surface, atrophy of adipose tissue in the synovial membrane, fibrosis in the lower layers of the synovial membrane, microvasculature expansion, and blood congestion. Furthermore, Evans et al. ${ }^{6)}$ and Yaoita ${ }^{7}$ investigated the effects of natural healing processes on these organic changes and reported that restrictions of range of motion and structural changes both recovered within a comparatively short time period. However, neither reported detailed histological findings, providing only a general overview.

This study aimed to investigate histopathological changes to clarify temporal effects on restrictions to range of motion and joint components after joint immobilization in a rat knee-contracture model.

\section{MATERIALS AND METHODS}

Fifty-four 8-week-old male Wistar rats (body weight 234-269 g) were used in this study. The rats were randomly divided into two groups: a fixation group $(\mathrm{n}=36)$ and a control group $(n=18)$. In the fixation group, right hindlimb knee joints were immobilized in full flexion by plaster casts, which were removed after four weeks. Subse- 
quent assessments were performed at weekly intervals. The research protocol followed Kanazawa University Animal Experiment Regulations and Kinjo University Experimental Animal Handling Regulations. All animal handling and experimentation were undertaken with the approval of the ethics committees of both universities (Kanazawa University: AP-091379 and Kinjo University: Approval no. 0003, 0004).

Control group rats were randomly subdivided into six groups. Rats from each group were raised in cages (similar to fixation group rats) up to ages corresponding to the subdivisions used for the fixation group. They were labeled as $0 \mathrm{WC}, 4 \mathrm{WFC}, 8 \mathrm{WFC}, 16 \mathrm{WFC}, 24 \mathrm{WFC}$, and $32 \mathrm{WFC}$, corresponding to the times of examination after cast removal: $0,4,8,16,24$, and 32 weeks, respectively.

Fixation group rats were immobilized with plaster casts according to a modified version of previously described methods $\left.^{10}, 12,13\right)$. Pentobarbital sodium $(5 \mathrm{mg} / 100 \mathrm{~g}$ body weight) anesthesia was administered through the abdominal cavity. Custom-made jackets produced from Velfoam (Velcro USA Inc., Manchester, NH, USA) were fitted to the animals' backs. To prevent scratches and abrasions to the right hind limb resulting from the plaster casts, the right hindlimb was covered with a layer of gauze that centered on the knee. The casts extended from the pelvic girdle to the ankle joint, with the hip joint at maximal extension, the knee joint at maximal flexion, and the ankle joint at maximal plantar flexion. The cast area was exposed from the distal ankle to the foot to confirm the absence of swelling and congestion. Considering the bone growth during the cast period, the kneecap was exposed as well.

Rats were able to freely move in their cages using their left hind limb and both forelimbs. Adequate levels of food and water were maintained during the raising period. If swelling was observed in the fixed limb, the fixation procedure was repeated, and casts were immediately replaced to prevent continuance. If casts were observed to slip out of place or become loose, they were also replaced as soon as possible. The cast period was set at four weeks on the basis of the findings of previous studies, which confirmed that histological changes were evident in the joint structure and content at this point.

Following the cast period, fixation group rats were randomly divided into six subgroups, with six rats in each group: these were labeled $0 \mathrm{WF}, 4 \mathrm{WF}, 8 \mathrm{WF}, 16 \mathrm{WF}, 24 \mathrm{WF}$, and $32 \mathrm{WF}$, corresponding to the examination times after cast removal: $0,4,8,16,24$, and 32 weeks, respectively. The OWF and $0 \mathrm{WC}$ groups underwent immediate assessments (described below) to measure the extension angle of their knee joints, to assess restrictions to range of motion, and to create histological specimens for analysis of the joints. Other groups were returned to their cages and raised under standard conditions. The restriction of knee joint extension angle was measured and recorded on the basis of a preliminary experiment. An intra-abdominal pentobarbital sodium anesthetic was administered ( $5 \mathrm{mg} / 100 \mathrm{~g}$ body weight), and the rats were laid on their sides. Using one hand to hold the femur in place, researchers used the other hand to hold the leg at $35 \mathrm{~mm}$ from the knee joint and guide the knee into maximal extension, the feeling experienced at the end of the range of motion. Using a digital camera, images of the principal axis of extension of the knee joint (the line running from the greater trochanter to the lateral condyle of the femur) and the movement axis (the line running from the head of the fibula to the lateral malleolus) were captured in the sagittal plane. The images were loaded onto a computer, and the angles of the principal and movement axes were measured using Three-Point Measuring Instrument software (Version 1.1.2). This protocol was used for all assessments. In addition, a digital force gauge (IMADA DPS-5) was attached at $35 \mathrm{~mm}$ from the knee joint, which was then extended to the angle measured. The force was recorded from the gauge. Three technicians, each with more than five years of clinical experience, performed each procedure five times. The average value was $0.33 \pm 0.05 \mathrm{~N}$. On the basis of these preliminary findings, all measurements of the restriction of knee joint extension angle were recorded by first attaching a digital force gauge at $35 \mathrm{~mm}$ from the knee joint, followed by application of $0.3 \mathrm{~N}$ of extension force and recording of the resulting angle. For the fixation group, these measurements were conducted before the experiment, immediately after cast removal and then weekly until the experiment was concluded. Measurements for the control group were conducted at the same intervals.

Individual differences in the development of contractures were assessed using retention rate calculations, which were as follows: retention rate $=[$ (the measured angle at each time-period) - (the angle at development of contracture) $] \times 100$.

At the end of each experimental period, a group of six animals was euthanized with a pentobarbital sodium overdose, and their hindlimbs were severed at the hip joint. Tissues were fixed over a 72 -h soaking period in a $10 \%$ buffered neutral formalin solution, and then decalcified using the Planck-Rychlo solution for $72 \mathrm{~h}$ at $4{ }^{\circ} \mathrm{C}$. Thereafter, an area approximately $10 \mathrm{~mm}$ above and below the knee joint was removed. Tissues from the center of the kneecap, moving toward its inner regions in the sagittal plane, were removed. To preserve this removed region, a 5-mm margin was cut and stored in an embedding cassette. It was subsequently neutralized in a 5\% anhydrous sodium sulfate solution for $72 \mathrm{~h}$, rinsed under running water for $30 \mathrm{~min}$, and soaked for approximately $3 \mathrm{~h}$ in $100 \%$ alcohol to remove any remaining fat. Thereafter, samples were processed in an automatic dehydrating-and-paraffin-wax-embedding device into paraffin blocks and sliced into $3-\mu \mathrm{m}$ thick sections using a sliding microtome (Leica SM2000R). After these organizational segments had been attached to a slide glass and dehydrated, they were stained with hematoxylin-eosin (HE) stain and enclosed in a hydrophobic mounting agent, to complete the histological specimen preparation. The specimens were observed using a microscopic digital camera (Olympus DP50) connected to an optical microscope (Olympus BX-51) to examine the entire knee joint structure. Although it is possible to classify cartilage on the basis of the degeneration it has undergone in cases of osteoarthritis $^{14-16)}$, histological classification of cartilage degeneration resulting from immobilization is a relatively new area of 
Table 1. Changes in knee extension angle restrictions

\begin{tabular}{lccccccc}
\hline & Immediate & \multicolumn{7}{c}{ Week following fixation removal } \\
\cline { 3 - 8 } & removal & 1th & 2nd & 3rd & 4th & 5th & 6th \\
\hline Group 4WF & $100 \%$ & $69.4 \pm 14.6 \%$ & $42.5 \pm 10.4 \%$ & $19.6 \pm 5.9 \%$ & $7.1 \pm 3.8 \%$ & & \\
Group 8WF & $100 \%$ & $74.4 \pm 5.9 \%$ & $50.5 \pm 4.8 \%$ & $20.3 \pm 8.5 \%$ & $7.8 \pm 5.3 \%$ & $1.4 \pm 1.1 \%$ & $0 \%$ \\
Group 16WF & $100 \%$ & $71.1 \pm 6.0 \%$ & $46.0 \pm 9.6 \%$ & $16.2 \pm 6.9 \%$ & $6.7 \pm 1.7 \%$ & $1.7 \pm 0.6 \%$ & $0 \%$ \\
Group 24WF & $100 \%$ & $76.6 \pm 8.8 \%$ & $41.4 \pm 8.4 \%$ & $16.0 \pm 4.5 \%$ & $7.5 \pm 3.2 \%$ & $0.5 \pm 1.1 \%$ & $0 \%$ \\
Group 32WF & $100 \%$ & $75.6 \pm 7.5 \%$ & $44.6 \pm 3.7 \%$ & $22.0 \pm 4.3 \%$ & $10.5 \pm 3.0 \%$ & $1.2 \pm 1.2 \%$ & $0 \%$ \\
\hline
\end{tabular}

Table 2. Abnormal findings in joint cartilage and the frontal synovial membrane

\begin{tabular}{lcccccc}
\hline & $\begin{array}{c}\text { Fibrosis of } \\
\text { cartilage }\end{array}$ & $\begin{array}{c}\text { Degeneration } \\
\text { of cartilage }\end{array}$ & $\begin{array}{c}\text { Thinning of } \\
\text { cartilage }\end{array}$ & $\begin{array}{c}\text { Adhesion of } \\
\text { frontal tibia }\end{array}$ & $\begin{array}{c}\text { Atrophy of } \\
\text { adipocyte }\end{array}$ & $\begin{array}{c}\text { Fibroblast } \\
\text { hyperplasia }\end{array}$ \\
\hline Group 0WF & 6 & 0 & 0 & 5 & 6 & 6 \\
Group 4WF & 6 & 0 & 0 & 3 & 6 & 6 \\
Group 8WF & 6 & 0 & 0 & 3 & 6 & 6 \\
Group 16WF & 6 & 2 & 0 & 3 & 6 & 6 \\
Group 24WF & 6 & 3 & 1 & 1 & 6 & 6 \\
Group 32WF & 6 & 5 & 2 & 0 & 6 & 6 \\
\hline
\end{tabular}

Numbers within the table indicate the number of specimens presenting the conditions out of a total of 6 .

study. Therefore, to quantify histological changes in the cartilage, we classified samples into one of the following four groups: Group I, fibrosis and degeneration could not be identified in outer layers; Group II, irregularities and fibrosis could be identified on the cartilage surface; Group III, thinning and transformation into fibrous pseudocartilage could be identified; and Group IV, destruction and damage could be identified in the cartilage.

For each group fitted with plaster casts, a comparative examination was conducted with the control group using Fisher's exact test using the statistical software, R (version 2.15.1). Significance was accepted for values less than 5\%.

\section{RESULTS}

Following the cast period, knee joint extension angles revealed following restrictions: $73.2 \pm 9.9^{\circ}$ in the $0 \mathrm{WF}$ group, $71.4 \pm 8.3^{\circ}$ in the $4 \mathrm{WF}$ group, $71.2 \pm 8.1^{\circ}$ in the $8 \mathrm{WF}$ group, $68.8 \pm 7.4^{\circ}$ in the $16 \mathrm{WF}$ group, $73.6 \pm 7.9^{\circ}$ in the $24 \mathrm{WF}$ group, and $70.2 \pm 11.2^{\circ}$ in the $32 \mathrm{WF}$ group. Using retention rate calculations, each group showed improvement over time, and the retention rate in the $4 \mathrm{WF}$ group was 7.1 $\pm 3.8 \%$ at the end of the study. Excluding the 4WF group, retention rates by the fifth week after cast removal were as follows: $8 \mathrm{WF}, 1.4 \pm 1.1 \%$; $16 \mathrm{WF}, 1.7 \pm 0.6 \%$; $24 \mathrm{WF}, 0.5 \pm$ $1.1 \%$; and $32 \mathrm{WF}, 1.2 \pm 1.2 \%$. Furthermore, retention rates of all groups were $0 \%$ by the sixth week. Knee extension angles were measured over the subsequent weeks, but none of the individual subjects in any group revealed any recurrence of their restrictions (Table 1).

Considering the relationship between age and histological changes, case-control comparisons matched by age produced the following data (Table 2, Fig. 1). In the articular cartilage of the 0WF group, fibrosis was identified on the surface of all cartilage specimens, and in five of six specimens, adhesion of the forward synovial membrane and fibrous structure of the hyperplasia was observed in the frontal tibial area. The gap between these adhesions resulted in narrowing of the articular cavity. All specimens revealed adipocyte tissue atrophy in the frontal synovial membrane, fibroblast hyperplasia in the lower layers, and expansion and blood congestion in the microvasculature. No significant changes were observed between $0 \mathrm{WF}, 4 \mathrm{WF}$, $8 \mathrm{WF}, 16 \mathrm{WF}, 24 \mathrm{WF}$ and $32 \mathrm{WF}$ groups in terms of adipocyte tissue atrophy and size, fibroblast hyperplasia, and microvasculature changes.

Although findings in the 4WF group were largely identical to those of the OWF group, adhesions were only found in three out of six samples. The same numbers of adhesions were observed in the $8 \mathrm{WF}$ group and the $4 \mathrm{WF}$ group, but the gap between fibers tended to become larger. Findings in the frontal synovial membrane were identical in $0 \mathrm{WF}$ and 4WF groups.

In addition, the results of articular cartilage examination in the $16 \mathrm{WF}$ were the same as for the $4 \mathrm{WF}$ and $8 \mathrm{WF}$ groups, but two of six specimens revealed replacement of the original hyaline cartilage with of a fibrous-type cartilage. Although adhesions of the frontal tibial area were identified in three of six specimens, gaps between fibers greater than those in the $8 \mathrm{WF}$ group, and fibrous structures were sparser.

Fibrosis of the articular cartilage surface was identified in all $24 \mathrm{WF}$ samples. However, only a partial adhesion was observed in the frontal tibial area of just one of six specimens. The gap between fibers was even greater than those observed in the 16WF group. In three of six samples, original cartilage was replaced with fibrous-type structures, and one of these revealed additional thinning in the cartilage.

Fibrosis of the articular cartilage but no adhesion was observed in the frontal tibial area in the articular cartilage 


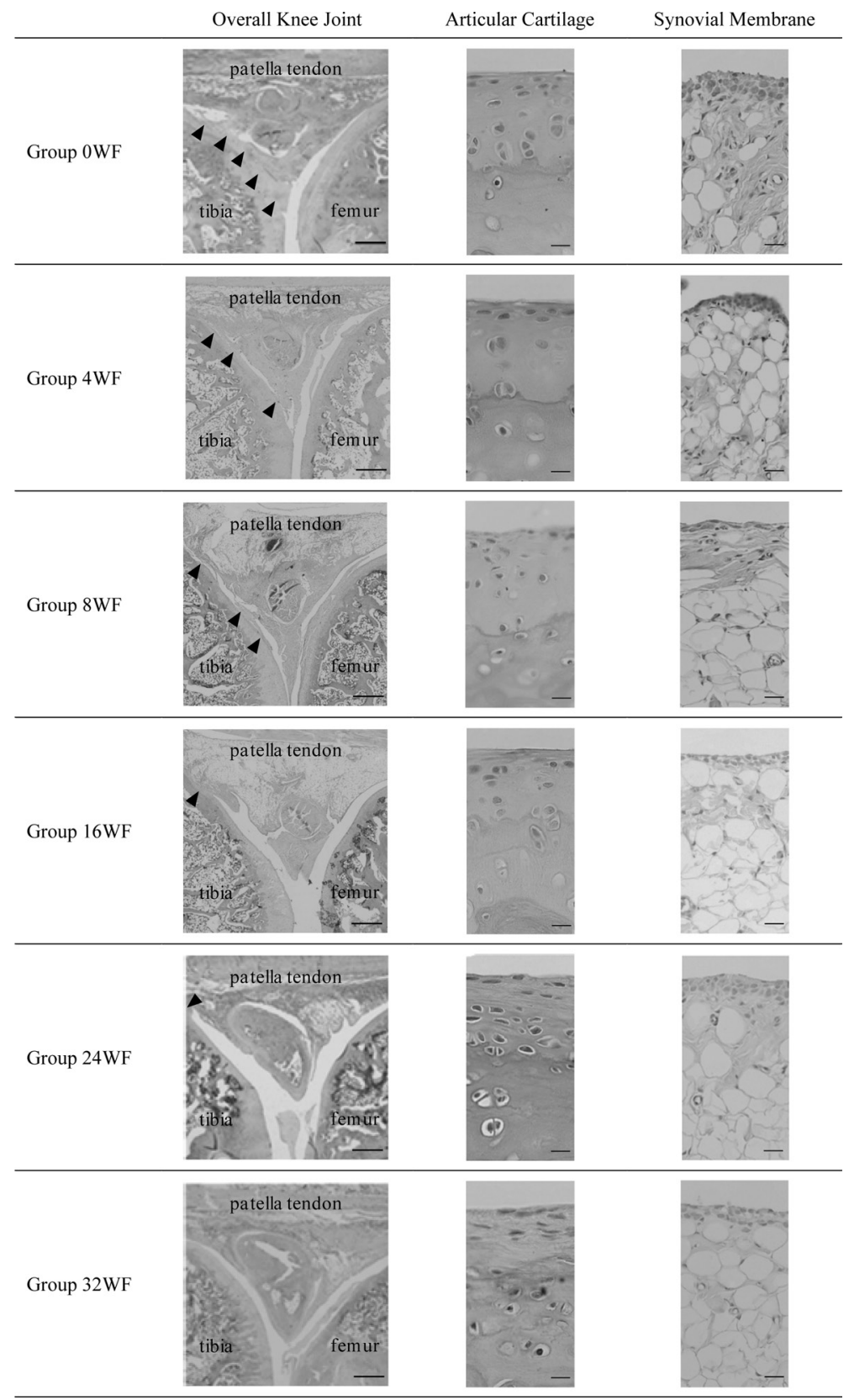

Fig. 1. Histological findings (HE stain, scale bar left: $1 \mathrm{~mm}$, center and right: $100 \mu \mathrm{m}$ )

The 0WF group: Adhesions of fibrous hyperplasia tissue between the frontal tibial area and the frontal synovial membrane were noted $(\boldsymbol{\Lambda}$, left). Fibrosis was identified in the articular cartilage surface (center). Adipocyte atrophy and fibroblast hyperplasia appeared, as well as expansion and blood congestion in the microvasculature (right). The 4WF group: Findings were the same as for the $0 \mathrm{WF}$ group. There were no clear differences in the extent of any of the conditions between the $4 \mathrm{WF}$ and $0 \mathrm{WF}$ groups. The $8 \mathrm{WF}$ group: Findings were the same as for the $0 \mathrm{WF}$ group. The space between adhesions was more expanded than in the $4 \mathrm{WF}$ group $(\boldsymbol{\Lambda}$, left). The 16WF group: Adhesions covered an even larger area than in the $8 \mathrm{WF}$ group (left). Some hyaline cartilage had been replaced with fibrous cartilage tissue (center). Findings related to conditions in the frontal synovial membrane were identical to those of the 0WF group (right). The $24 \mathrm{WF}$ group: Spaces between adhesions were bigger than in the 16WF group ( $\boldsymbol{\Lambda}$, left). Cartilage degeneration and thinning were identified (center). Findings related to conditions in the frontal synovial membrane were identical to those of the 0WF group (right). The 32WF group: No adhesions were evident (left). Cartilage degeneration and thinning were identified (center). Findings related to conditions in the frontal synovial membrane were identical to those of the 0WF group (right). 
samples of the 32WF group. However, the original cartilage was replaced with fibrous-type structures in five of six cases, and the degeneration was quite severe; two of these revealed additional thinning the cartilage. All adhesions had disappeared in this group.

Finally, inflammatory cell infiltration was observed in all groups. On the basis of histological classification of the cartilage in the cast group compared with the control group, a significant difference was observed between both groups (Table 3, p < 0.05).

\section{DISCUSSION}

In this study, six weeks after cast removal, all rats showed complete recovery in their range of knee joint extension. However, although the degree of contracture-associated adhesions decreased over time, a replacement of existing cartilage with fibrous-type structures along with thinning of the cartilage was observed. Moreover, at all stages, there were persistent changes in the frontal synovial membrane, atrophy and differences in the size of adipocyte tissues, fibroblast hyperplasia in lower layers, and expansion and blood congestion in the microvasculature. Therefore, although the range of knee joint extension improved, the articular cartilage and frontal synovial membrane remained damaged.

Evans et al. ${ }^{6}$ evaluated the restoration of mobility in immobilized joints following the onset of contracture using an internal-fixation knee-arthrodesis contracture model in rats. Their study reported that provided, the fixation lasted no longer than 30 days, changes in the range of motion were reversible. Yaoita ${ }^{7)}$ and Maoka $^{17)}$ also reported that range of motion and organizational structures were restored comparatively quickly following short-term fixation of 30 days or less in a rat knee contracture model. Although we found some differences in the time required to restore mobility and used a different fixation method, the complete restoration of mobility we observed agrees with prior research.

The hyaline cartilage forms a structure around joints and functions as a load-bearing buffer, reducing friction between bones in the course of normal joint movement, and playing a role in maintaining mobility. Hyaline cartilage does not contain blood cells, lymph ducts, or nerves, and does not directly experience inflammation ${ }^{18)}$.

In a broader evaluation of joint changes resulting from contracture, Evans et al. ${ }^{6}$ examined histological changes in rats with internal fixation knee arthrodeses. They reported that after 30 days of fixation, there was a proliferation of intra-articular connective tissue as well as fibrosis and blood congestion under the kneecap and in the intercondylar fossa. They also observed cartilage compression at contact points of the femur and tibial condyles, and miniaturization of chondrocytes, and they concluded that intra-articular connective tissue hyperplasia and adhesions within the cartilage were the cause of the restricted range of motion. These adhesions did not completely cover the cruciate ligament of the knee and its surroundings, rather they originated from fibrosis of the synovial membrane.

Similar to results of the study conducted by Evans et
Table 3. Histological classification of cartilage

\begin{tabular}{lcc}
\hline & $\begin{array}{c}\text { Group } \\
\text { OWF }\end{array}$ & $\begin{array}{c}\text { Group } \\
\text { 0WC }\end{array}$ \\
\hline I & 0 & 3 \\
II & 6 & 0 \\
III & 0 & 0 \\
IV & 0 & 0 \\
\hline & & $\mathrm{p}=0.011$ \\
& & \\
\hline & Group & Group \\
& 8 WF & 8 WFC \\
\hline I & 0 & 3 \\
II & 6 & 0 \\
III & 0 & 0 \\
IV & 0 & 0 \\
\hline & & $\mathrm{p}=0.011$ \\
& & \\
\hline & Group & Group \\
& $24 W F$ & $24 W F C$ \\
\hline I & 0 & 3 \\
II & 3 & 0 \\
III & 3 & 0 \\
IV & 0 & 0 \\
\hline & & $\mathrm{p}=0.036$
\end{tabular}

\begin{tabular}{lcc}
\hline & $\begin{array}{c}\text { Group } \\
4 \mathrm{WF}\end{array}$ & $\begin{array}{c}\text { Group } \\
4 \mathrm{WFC}\end{array}$ \\
\hline I & 0 & 3 \\
II & 6 & 0 \\
III & 0 & 0 \\
IV & 0 & 0 \\
\hline & & $\mathrm{p}=0.011$ \\
& & \\
\hline & Group & Group \\
& $16 \mathrm{WF}$ & $16 \mathrm{WFC}$ \\
\hline I & 0 & 3 \\
II & 4 & 0 \\
III & 2 & 0 \\
IV & 0 & 0 \\
\hline & & $\mathrm{p}=0.011$ \\
& & \\
\hline & Group & Group \\
& $32 \mathrm{WF}$ & $32 \mathrm{WFC}$ \\
\hline I & 0 & 3 \\
II & 1 & 0 \\
III & 5 & 0 \\
IV & 0 & 0 \\
\hline & & $\mathrm{p}=0.034$
\end{tabular}

Numbers indicate the number of confirmed specimens.

al. ${ }^{6)}$, Yaoita et al. ${ }^{7)}$, using internal fixation for the knee joint in rats, revealed thinning of cartilage tissue, disordered cell arrangement, and necrobiosis after 30 days of fixation. Because of these structural changes, fibrous connective tissues and adipocytes filled the intra-articular area from the rear of the joint, extending the joint surface forward, parallel to the femur, narrowing the articular space. Both the studies cited here discussed the process used to induce contracture in detail and highlighted resulting histological changes. However, their descriptions of the recovery process after the removal of fixation was limited to general statements about the range of motion being restored to its pre-experimental levels, and the joint being otherwise normal.

We could find no other study in the literature which described the progress of contracture and histological joint changes over time. Moreover, the hyperplasia we observed in the membrane tissue of the outer layer of articular cartilage is consistent with our previously published reports ${ }^{10-13)}$ and could have resulted from joint immobilization. In addition, adhesions within the frontal synovial membrane and the frontal tibial area were consistent with the findings of Watanabe et al. ${ }^{10)}$ during long-term cast fixation. Although these adhesions may be consistent with the work of Evans et al. and Yaoita, the position in which the adhesions developed were clearly different. This could be explained by differences in the fixation methods and observation areas among the studies, but the true reasons remain unclear and require further investigation.

In the present study, as the time interval progressed, adhesions diminished; moreover, by 32 weeks after cast re- 
moval, they had completely disappeared. This agrees with the findings of Evans et al. and Yaoita, who noted that free movement after removal of fixation resulted in mechanical stress, though it is equally possible that the adhesions may have simply detached. In contrast with the resolution of adhesions, the number of cases, wherein normal cartilage was replaced with fibrous structures and thinning, increased as time progressed.

Reports of thinning and degeneration of cartilage have been extremely rare until now. Ely et al. ${ }^{8}$ evaluated changes in joint components resulting from cast fixation in adult dogs, and reported a narrowing of the space between opposite cartilage structures along with thinning of the cartilage in load-bearing areas. In addition, Hagiwara et al. ${ }^{19)}$ evaluated knee articular cartilage thickness in rats after internal fixation at $150^{\circ}$, and reported that the thickness increased in movement areas, although it remained unchanged in contact and non-contact areas. The timing of the appearance of cartilage thinning in the study of Hagiwara et al. clearly differs from that reported by Ely et al. and Yaoita. Furthermore, the cartilage thickening observed by Hagiwara et al. was not observed in any sample in the present study.

We offer two hypotheses as to why these differences occurred. First, because adhesions connect viscera and tissue that should be separated ${ }^{20)}$, the detachment of these adhesions through mechanical stress may have caused bleeding and inflammation ${ }^{21)}$. As a result, the normal repair process may have caused the replacement of normal cartilage with fibrous tissue structures. Second, the articular cartilage derives nutrition from synovial fluid, and if this nutritional supply were obstructed by surface tissue hyperplasia, it might cause degeneration. This could then result in a decline in cartilage regeneration, necrobiosis, and decreasing thickness, resulting in the replacement of cartilage with fibrous tissues through the recovery process ${ }^{21)}$. These qualitative changes to the articular cartilage did not occur immediately after the onset of the contracture but after an amount of time had passed. This cartilage degeneration is a new finding, and future biochemical analyses will be required to clarify its causes.

Takemura et al. ${ }^{12)}$ analyzed changes in the frontal synovial membrane resulting from contracture with regard to contractures induced by cast fixation. Joint immobilization resulted in adipocyte tissue atrophy and size differences in the frontal synovial membrane, fibroblast hyperplasia in the lower layers, and expansion and blood congestion in the microvasculature. Watanabe et al. ${ }^{10)}$ and Matsuzaki et al. ${ }^{11)}$ reported identical findings, and these were unsurprisingly confirmed by our study. These changes may have occurred because of a decrease in the mechanical stress resulting from the contracture.

To date, very few articles have prospectively considered the recovery process of the frontal synovial membrane following immobilization. Although Evans et al. and Yaoita provided detailed descriptions of the recovery process, they only made general statements about histological improvement. Conversely, Takemura et al. and Matsuzaki et al. failed to observe any improvement in frontal synovial membrane atrophy. In this study, we observed adipocyte tissue atrophy and size differences in the frontal synovial membrane, fibroblast hyperplasia in the lower layers, and expansion and blood congestion in the microvasculature. Moreover, this occurred at all the examination periods (up to and including 32 weeks after removal of cast fixation). Even after the passage of a long time interval, no trends toward improvement were identified in the findings. To the best of our knowledge, although articles have reported the posterior synovial membrane changes that result from joint immobilization, our report is the first to observe atrophy of adipose tissue in the frontal synovial membrane. In addition, atrophy of structural fat such as the corpus adiposum infrapatellar as well as bone marrow is not thought to easily occur in such cases ${ }^{11)}$. Therefore, future histological and biochemical investigations may be necessary to clarify how this occurs.

The results of our present study are in agreement with previous research that joint mobility may completely recover after contracture. We further add that this may occur through time alone, and that damage to the articular cartilage and frontal synovial membrane did not improve within 32 weeks after fixation, the end of our observation period. Briefly, free motion merely improves mobility and does not result in any organic improvements in the joint itself. When physical therapists passively mobilize joints to improve range of motion following contractures, they often experience a different sensation toward the end of the range of motion. This may result from an increased coefficient of friction because of the fibrosis and degeneration in the cartilage surface. The inadequate consideration of these issues in reports to date means future research focusing more closely on appropriate medical interventions to limit organic changes is required. Furthermore, future research must respond to advances in our understanding of the fundamental scientific basis of physical therapy.

\section{ACKNOWLEDGEMENTS}

The authors would like to express their heartfelt gratitude for the degree of guidance and cooperation we received from many professors at the Department of Morpho-Functional Pathology, Division of Cancer Medicine, Graduate School of Medical Sciences, Kanazawa University. This work was supported by Grants-in-Aid for Scientific Research (KAKENHI) from the Japan Society for the Promotion of Science (25750229).

\section{REFERENCES}

1) Takemasa $\mathrm{K}$, Shimada T, Hidaka M: Normal range of motion of joints in the aged people. Kobe Daigaku Igakubu Hoken Gakka Kiyo, 1997, 1997: 77-82 (in Japanese)

2) Koizumi K, Hoso M, Hamade S, et al: Prevention and Treatment of Contracture, 2nd ed. Tokyo: Igakusyoin, 2008, pp 1-17, 19-22, 67-77 (in Japanese).

3) Abe T: Rigaku Ryoho Hyokagaku, 2nd ed. Tokyo: Igakusyoin, 2004, pp 76-92 (in Japanese).

4) Neumann DA: Kinesiology of the Musculoskeletal System. St. Louis: Mosby, 2002, pp 25-40.

5) Grabois M, Garrison SJ, Hart KA, et al.: Physical Medicine \& Rehabilitation. The Complete Approach. Massachusetts, Blackwell Science Inc., 2000, pp 859-870. 
6) Evans EB, Eggers GW, James K, et al.: Experimental immobilization and remobilization of rat knee joints. J Bone Joint Surg. 1960, 42A: 737-758.

7) Yaoita M: Effect of immobilization and subsenquent remobilization of the knee joint of the rats. J Jap Orthop Ass. 1966, 40: 431-453 (in Japanese).

8) Ely LW, Mensor MC: Studies on the immobilization of the normal joints. Surg Gynecol Obstet, 1933, 57: 212-215.

9) Trudel G, Uhthoff HK: Contractures secondary to immobility: is the restriction articular or muscular? An experimental longitudinal study in the rat knee. Arch Phys Med Rehabil, 2000, 81: 6-13. [Medline]

10) Watanabe $M$, Hoso $M$, Takemura $K$, et al.: Histopathological changes in joint components during contracture - A study of the long term knee joint immobility model in the rat-. Rigakuryoho Kagaku, 2007, 22: 67-75 (in Japanese). [CrossRef]

11) Matsuzaki T, Kojima S, Watanabe M, et. al.: Histopathological changes in the infrapatellar fat-pad of rats with knee contracture. Ishikawa Journal of Physical Therapy, 2012, 12: 11-14 (in Japanese).

12) Takemura K, Hoso M, Yoshikubo H, et al.: Histopathological Effects of the Stretching on Joint Components after Two-week Knee Joint Immobilization in Rats. Rigakuryohogaku, 2004, 31: 76-85 (in Japanese).

13) Kojima $S$, Hoso M, Takemura K, et al.: Histopathological effect of the hot bath on joint components knee joint immobilization in rats. Rigakuryoho Kagaku, 2009, 24: 161-166 (in Japanese). [CrossRef]
14) Pritzker KP, Gay S, Jimenez SA, et al.: Osteoarthritis cartilage histopathology: grading and staging. Osteoarthritis Cartilage, 2006, 14: 13-29. [Medline] [CrossRef]

15) Aigner T, Söder S: [Histopathological examination of joint degeneration: typing, grading and staging of osteoarthritis]. Pathologe, 2006, 27: 431438. [Medline] [CrossRef]

16) Mankin HJ, Dorfman H, Lippiello L, et al.: Biochemical and metabolic abnormalities in articular cartilage from osteo-arthritic human hips. II. Correlation of morphology with biochemical and metabolic data. J Bone Joint Surg Am, 1971, 53: 523-537. [Medline]

17) Maoka N: An expcrimental study of functional rccovery in stiffened knee joints. Shikoku Igaku Zasshi, 1964, 20: 38-51 (in Japanese).

18) Okita M, Nakano J, Sakai T, et al.: Limited Range of Joint Motion. Tokyo: Miwasyoten, 2008, pp 2-17, 70-88, 126-150 (in Japanese).

19) Hagiwara $Y$, Ando A, Chimoto E, et al.: Changes of articular cartilage after immobilization in a rat knee contracture model. J Orthop Res, 2009, 27: 236-242. [Medline] [CrossRef]

20) Fujita K: Saishin Igaku Daijiten, 3rd ed. Tokyo: Ishiyakusyuppan, 2005, p 1858 (in Japanese).

21) Yoshikawa H: Lesion Repair and Regeneration of Bone/Cartilage. Hyojun Seikei Gekagaku, 11th ed. Tokyo: Igakusyoin, 2011, pp 53-60 (in Japanese). 